\title{
Some new ideas for a Schiffer's conjecture
}

\author{
T. Chatelain, M. Choulli and A. Henrot \\ Équipe de Mathématiques - URA CNRS 741 \\ Université de Franche-Comté, F-25030 Besançon Cedex France
}

\begin{abstract}
We discuss a Schiffer's conjecture which is a symmetry problem for an overdetermined spectral p.d.e.. We show the connection between this problem and the critical points of the eigenvalue with a volume constraint as well as the Faber-Krahn inequality. We give two original proofs of these symmetry results in the case of the first eigenvalue.
\end{abstract}

\section{Keywords}

Domain derivative, first eigenvalue, Faber-Krahn inequality, continuous Steiner symmetrization

\section{INTRODUCTION}

The motivation of this work is a conjecture of M. Schiffer in spectral theory: let $\Omega$ be a connected regular domain in $\mathbf{R}^{N}$ such that there exists an eigenvalue $\lambda$ and a Dirichleteigenfunction $u \neq 0$ satisfying

$$
\left\{\begin{array}{ccccc}
-\Delta u & = & \lambda u & \text { in } & \Omega \\
u & = & 0 & \text { on } & \partial \Omega \\
\frac{\partial u}{\partial n} & = & c s t & \text { on } & \partial \Omega
\end{array}\right.
$$

then does it imply that $\Omega$ is a ball ?

A domain $\Omega$ for which $(S C)$ has a non trivial solution will be called in the following a solution of the $(S C)$ problem.

This conjecture, for the Dirichlet case, can also, of course, be considered for the Neumann case : if $\lambda>0$ and $u \neq 0$ are such that

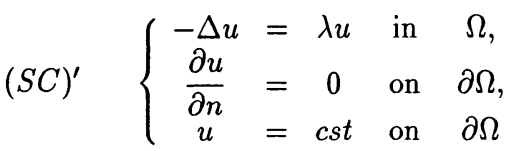

This conjecture $(S C)^{\prime}$ has been more intensively studied than the previous one, because 
Williams in a paper of 1976 , proves that it was equivalent to the famous Pompeiu problem. In spite of many efforts these twenty last years, only partial results are available for $(S C)^{\prime}$, a good reference is Zalcman(1992), and (to our knowledge), very few is known for $(S C)$. So these two conjectures $(S C)$ and $(S C)^{\prime}$ remain open even in 2-dimensions.

In this paper, we are interested in $(S C)$ for the case $\lambda=\lambda_{1}$ the first Dirichlet eigenvalue. Of course, in this case the result is already known, since it is a consequence for example of the classical result of Serrin (1971). Nevertheless, our aim is to give another original proof of this result which does not use maximum principle.

This fact is of importance since maximum principle is unavailable for $(S C)$ when $\lambda \geq \lambda_{2}$ and for $(S C)^{\prime}$. Unfortunately, we apparently need in our approach the positivity of the eigenfunction. We also link this Schiffer's conjecture, using domain derivative, to the classical problem of minimizing the first eigenvalue with a volume constraint (see also Cox (1994) for similar ideas) and we give another proof of the fact that equality in the Faber-Krahn inequality is achieved only for the ball.

Some of the ideas developed in this paper could be used for the conjecture $(S C)$ or $(S C)^{\prime}$ in the general case. A work in this direction is in preparation (see Chatelain and Henrot(work in progress)).

Let us describe now briefly the content of this paper.

In the second part, we recall the classical formula of derivative of the first eigenvalue with respect to the domain. This formula allows us to point out the link which exists between the Schiffer's conjecture $(S C)$, the isoperimetric Faber-Krahn inequality and the critical point of the first eigenvalue, considered as a domain functional, under a volume constraint. Indeed we make the simple observation that $\Omega$ is a solution of the $(S C)$ problem if and only if there exists a constant $c$ such that

$d \lambda(\Omega ; V)=c d V o l(\Omega ; V)$ for all displacement field $V$,

where $d \lambda(\Omega ; V)$ and $d V o l(\Omega ; V)$ denote respectively the derivative of $\lambda$ and the derivative of the volume at the point $\Omega$ in the direction of the displacement field $V$.

In the third part, we prove that equality in the Faber-Krahn inequality is realized only for balls. In other words, the only minimum for the first eigenvalue with a volume constraint is the ball. This result was, of course, already known ; but as pointed out in Kawohl (1985), most of the proofs were done in the analytic case and used the analyticity of the eigenfunction.

At last, in the fourth part, we prove, using a continuous Steiner symmetrization, that, if $\Omega$ is a convex domain which is not a ball, there exists at least one displacement field $V$ such that (1) fails. This will imply the desired result that the only solution (at least in the convex case) of $(S C)$ are the balls. The same idea is used in a similar context in Brock and Henrot (work in progress) to prove a generalization of the Serrin's result to some nonlinear elliptic problems.

\section{DERIVATIVE OF THE FIRST EIGENVALUE AND SOME CONSEQUENCES}

Domain derivative is now a very usual tool in field such as shape optimization. From a mathematical point of view, it goes from Hadamard in 1905 and Garabedian-Schiffer 
in 1953. Good references are the works of Murat-Simon in 1975 which is summed up in a paper of Simon (1980), or the more recent book of Sokolowski and Zolesio (1992).

The first eigenvalue of the Laplacien-Dirichlet, $\lambda_{1}(\Omega)$ which is characterized as

$$
\lambda_{1}(\Omega)=\inf _{\substack{v \in H_{0}^{1}(\Omega) \\ v \neq 0}} \frac{\int_{\Omega}|\nabla v|^{2}}{\int_{\Omega} v^{2}}
$$

can be regarded as a domain functional since it depends obviously on the domain $\Omega$. Moreover, for a connected domain, $\lambda_{1}(\Omega)$ is a simple eigenvalue so it is possible to prove that $\Omega \mapsto \lambda_{1}(\Omega)$ is differentiable. This is proved, for example in Rousselet and Chesnais (1990), for a similar problem in Mignot, Murat and Puel (1979) and also formally (from a "mechanist" point of view) in Sanchez-Hubert and Sanchez-Palencia. We do not want, here, to give the proof of the differentiability. It is technical and it uses the Fredholm alternative and an implicit function theorem. Let us just establish here the formula of the derivative of the first eigenvalue by a formal calculation : we assume in the following that $\Omega$ is a $C^{2}$ connected bounded domain and let $u(=u(\Omega))$ be a positive normalized eigenfunction associated to $\lambda_{1}$. In the sequel we will drop out the subscript 1 since we are always working with the first eigenvalue, so $\lambda=\lambda_{1}$ and :

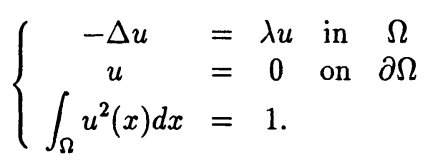

Let us choose a field of deformation $V \in C^{2}\left(\mathbf{R}^{N} ; \mathbf{R}^{N}\right)$. By differentiating (3), it is well known that $u^{\prime}$ (the derivative of $u$ with respect to the domain in the direction of $V$ ) satisfies

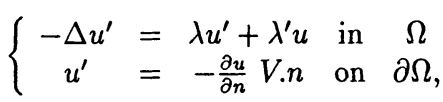

where $\lambda^{\prime}$ is the derivative of $\lambda$ with respect to the deformation of $\Omega$ by $\mathrm{V}$. Multiplying the first equation by $\mathrm{u}$ and integrating on $\Omega$ yields

$-\int_{\Omega} u \Delta u^{\prime} d x=\lambda \int_{\Omega} u u^{\prime} d x+\lambda^{\prime} \int_{\Omega} u^{2} d x=\lambda \int_{\Omega} u u^{\prime} d x+\lambda^{\prime}$

but the Green formula gives

$-\int_{\Omega} u \Delta u^{\prime} d x=-\int_{\partial \Omega} u \frac{\partial u^{\prime}}{\partial n} d \sigma+\int_{\partial \Omega} u^{\prime} \frac{\partial u}{\partial n} d \sigma+\lambda \int_{\Omega} u^{\prime} u d x$

so using (3) and (4) and replacing in (5) yields the desired formula :

$\lambda^{\prime}=d \lambda(\Omega ; V)=-\int_{\partial \Omega}\left(\frac{\partial u}{\partial n}\right)^{2} V \cdot n d \sigma$. 
Since the derivative of the volume is given by

$d V o l(\Omega ; V)=\int_{\partial \Omega} V \cdot n d \sigma$

we have immediately

Proposition $1 \Omega$ is a solution of the problem $(S C)$ if and only if there exists a constant c such that

$d \lambda(\Omega ; V)=-c^{2} d V o l(\Omega ; V)$.

In other words, the solutions of the problem $(S C)$ can be considered as critical points of the functional $\lambda(\Omega)$ with a volume constraint, the constant $-c^{2}$ being a Lagrange multiplier.

Another consequence of the proposition 1 is the following : the well known Faber-Krahn tells us that for all domains $\Omega$ with $\operatorname{Vol}(\Omega)=V_{0}$ given, we have

$\lambda_{1}\left(\Omega^{*}\right) \leq \lambda_{1}(\Omega)$

where $\Omega^{*}$ is the ball of volume $V_{0}$. Now, thanks to proposition 1 , any such minimum satisfies (9) and then

Corollary $1 \Omega$ realizes the equality in the Faber-Krahn inequality if and only if $\Omega$ is a solution of the problem $(S C)$.

As an application of the Serrin's result (Serrin, 1971) we obtain immediately that :

Theorem 1 (equality in the Faber-Krahn inequality) Let

$\Theta=\left\{\Omega\right.$ bounded, connected $C^{2}$ domains in $\left.\mathbf{R}^{N}, \operatorname{Vol}(\Omega)=V_{0}\right\}$

and $\Omega^{*}$ be the ball of volume $V_{0}$ then

$\lambda_{1}(\Omega)=\lambda_{1}\left(\Omega^{*}\right)$ and $\Omega \in \Theta$ if and only if $\Omega=\Omega^{*}$.

\section{ANOTHER (ELEMENTARY) PROOF OF THEOREM 1}

The aim of this section is to give another very simple proof of the theorem 1 :

let $\Omega$ be a minimum of $\lambda_{1}$ with $\operatorname{Vol}(\Omega)=V_{0}$ (that is to say a domain for which we have equality in the Faber-Krahn inequality). According to corollary 1, we have

$\frac{\partial u}{\partial n}=$ constant $=c \quad$ on $\partial \Omega$

and then, integrating over $\partial \Omega$ gives :

$c P(\partial \Omega)=\int_{\partial \Omega} \frac{\partial u}{\partial n} d \sigma=\int_{\Omega} \Delta u d x=-\lambda(\Omega) \int_{\Omega} u d x$, 
where $P(\Omega)$ is the perimeter of $\partial \Omega$.

Now we use the well known Rellich identity (see Rellich (1940)) :

$2 \int_{\partial \Omega}(x . \nabla u) \frac{\partial u}{\partial n} d \sigma-\int_{\partial \Omega}(x . n)|\nabla u|^{2} d \sigma=2 \int_{\Omega}(x . \nabla u) \Delta u d x+(2-N) \int_{\Omega}|\nabla u|^{2} d x$.

In our case, this identity gives :

$2 \lambda(\Omega)=2 \lambda(\Omega) \int_{\Omega} u^{2} d x=N c^{2} \operatorname{Vol}(\Omega)$

and then (12) and (14) yield

$\lambda(\Omega)\left(\int_{\Omega} u d x\right)^{2}=\frac{2 P(\partial \Omega)^{2}}{N \operatorname{Vol}(\Omega)}$.

Now, let us introduce $u^{*}$ the (Schwarz) spherical decreasing rearrangement of $u$ (see Kawohl (1985)) which is defined on the ball $\Omega^{*}$. We know that

$\int_{\Omega^{*}} u^{*^{2}}(x) d x=\int_{\Omega} u^{2}(x) d x=1$

that

$\int_{\Omega^{*}} u^{*}(x) d x=\int_{\Omega} u(x) d x$

and also that (Polya-Szegö theorem)

$\int_{\Omega^{*}}\left|\nabla u^{*}(x)\right|^{2} d x \leq \int_{\Omega}|\nabla u(x)|^{2} d x=\lambda_{1}(\Omega)$.

Now, by (2) and (16) :

$\lambda(\Omega)=\lambda\left(\Omega^{*}\right) \leq \int_{\Omega^{*}}\left|\nabla u^{*}(x)\right|^{2} d x \leq \int_{\Omega}|\nabla u(x)|^{2} d x=\lambda(\Omega)$.

Then, the inequality chain in (17) is an equality chain and

$\lambda\left(\Omega^{*}\right)=\int_{\Omega^{*}}\left|\nabla u^{*}(x)\right|^{2} d x$,

which implies that $u^{*}$ is a (normalized) eigenfunction associated to $\lambda\left(\Omega^{*}\right)$. Now identity (15) remains true for $u^{*}$ and $\Omega^{*}\left(u^{*}\right.$ being radial, its normal derivative is constant and then all the calculations are valid). So as a consequence :

$\frac{2 P(\partial \Omega)^{2}}{N \operatorname{Vol}(\Omega)}=\lambda(\Omega)\left(\int_{\Omega} u d x\right)^{2}=\lambda\left(\Omega^{*}\right)\left(\int_{\Omega^{*}} u^{*} d x\right)^{2}=\frac{2 P\left(\partial \Omega^{*}\right)^{2}}{N \operatorname{Vol}\left(\Omega^{*}\right)}$.

Now, since $\operatorname{Vol}\left(\Omega^{*}\right)=\operatorname{Vol}(\Omega),(18)$ implies that $P\left(\partial \Omega^{*}\right)=P(\partial \Omega)$ and the classical isoperimetric inequality gives us the desired result. 


\section{CONTINUOUS STEINER SYMMETRIZATION}

In this section, we want to give another proof (in a particular case) of the Serrin's theorem for the problem $(S C)$, which is based on the proposition 1 and which does not use maximum principle.

Theorem 2 The only convex solutions of the problem $(S C)\left(\right.$ for $\left.\lambda=\lambda_{1}\right)$ are the balls.

Our proof is based on the following very simple idea. Since each solution of the problem $(S C)$ satisfies (9), to prove theorem 2 it is sufficient to prove that for a domain $\Omega$ which is not a ball, there exists (at least) one vector field of displacement $V$ such that (9) is false. More precisely, we are going to exhibit a vector field of displacement $V$ (given by the Continuous Steiner symmetrization) which leaves the volume unchanged, then $d V o l(\Omega ; V)=0$, and such that

$d \lambda(\Omega ; V)<0$,

which will give the theorem 2. The use of a continuous Steiner symmetrization field of displacement can also be found in Brock and Henrot (to appear) to prove a more general result.

Let us assume that $\Omega$ is not a ball : so there exists a (direction of) hyperplane with respect to which $\Omega$ is not symmetric. Let us denote by $\left(x^{\prime}, y\right), x^{\prime} \in \mathbf{R}^{N-1}, y \in \mathbf{R}$, the points in $\mathbf{R}^{N}$, and we can always assume that the hyperplane is $y=0$. Since $\Omega$ is convex, the intersection of $\Omega$ with every line $x^{\prime}=$ constant is a segment say $y_{1}\left(x^{\prime}\right) \leq y_{2}\left(x^{\prime}\right)$.

Then we define a displacement field $V: \bar{\Omega} \rightarrow \mathbf{R}^{N}$ by :

$V\left(x^{\prime}, y\right)=\left(0,-\frac{1}{2}\left(y_{1}\left(x^{\prime}\right)+y_{2}\left(x^{\prime}\right)\right)\right)$

(where 0 is the zero of $\mathbf{R}^{N-1}$ ) which is continuously extended to $\mathbf{R}^{N}$.

In this way $\Omega_{t}=(\mathrm{Id}+\mathrm{t} V)(\Omega)$ is the classical continuous Steiner symmetrization of $\Omega$ (see Brock(1995)) and we know that, for every positive continuous function $f$ in the Sobolev space $H_{0}^{1}(\Omega)$, we are able to define $f^{t}$, its continuous Steiner symmetrization and we have

$\int_{\Omega_{t}} f^{t^{2}}(x) d x=\int_{\Omega} f^{2}(x) d x$

$\int_{\Omega_{t}}\left|\nabla f^{t}(x)\right|^{2} d x \leq \int_{\Omega}|\nabla f(x)|^{2} d x$.

The following lemma gives a more precise statement of the inequality (22) in the case of a quasiconcave function $f$ (i.e. a function $f$ whose level sets are convex) :

Lemma 1 Let $u$ be a quasiconcave function as above defined on the convex set $\Omega$ and $u^{t}$ its continuous Steiner symmetrization; then when $t$ goes to zero we have the expansion

$\int_{\Omega_{t}}\left|\nabla u^{t}(x)\right|^{2} d x=\int_{\Omega}|\nabla u(x)|^{2} d x+\alpha(u, \Omega) t+o(t)$

with $\alpha(u, \Omega)$ (which is a number depending on $u$ and $\Omega$ ) strictly negative as soon as $\Omega$ is not symmetric with respect to $\{y=0\}$. 
Proof. Let us denote by

$\Delta=\left\{\left(x^{\prime}, u\right) \in \mathbf{R}^{N-1} \times \mathbf{R}_{+} / \exists\left(x^{\prime}, y\right) \in \Omega\right.$ such that $\left.u=u\left(x^{\prime}, y\right)\right\}$

The level sets of $u$ being convex, for each $\left(x^{\prime}, u\right) \in \Delta$ the equation $u=u\left(x^{\prime}, y\right)$ has exactly two solutions $y=y_{1}$ and $y=y_{2}$ with $y_{1} \leq y_{2}$. Thus $u$ can be represented in $\Delta$ by the inverse function $y=y_{k}\left(x^{\prime}, u\right), k=1,2$ and moreover we have

$u_{y}\left(x^{\prime}, y_{k}\right)=\left(\frac{\partial y_{k}}{\partial u}\right)^{-1} \begin{cases}>0 & \text { for } k=1 \text { (almost everywhere) } \\ <0 & \text { for } k=2 \text { (almost everywhere) }\end{cases}$

$u_{x_{i}}\left(x^{\prime}, y_{k}\right)=-\frac{\partial y_{k}}{\partial x_{i}}\left(\frac{\partial y_{k}}{\partial u}\right)^{-1} \quad(i=1, \ldots, N-1)$.

At last, by definition of the continuous symmetrization (see Brock, 1995), $u^{t}$ is defined by the inverse functions $y_{k}^{t}, k=1,2$ given by

$y_{k}^{t}=y_{k}-\frac{t}{2}\left(y_{1}+y_{2}\right) \quad k=1,2$.

By the change of variable induced by $y_{k}$, we have

$$
\int_{\Omega_{t}}\left|\nabla u^{t}(x)\right|^{2} d x=\int_{\Delta} \sum_{k=1}^{2}\left|\frac{\partial y_{k}^{t}}{\partial u}\right|^{-2}\left(1+\sum_{i=1}^{N-1}\left(\frac{\partial y_{k}^{t}}{\partial x_{i}}\right)^{2}\right)\left|\frac{\partial y_{k}^{t}}{\partial u}\right| d x^{\prime} d u
$$

this formula remaining valid for $t=0$. Using (26) and a straightforward calculation, we obtain immediately

$$
\begin{aligned}
& \int_{\Omega_{t}}\left|\nabla u^{t}(x)\right|^{2} d x=\int_{\Omega}|\nabla u(x)|^{2} d x \\
& +t \sum_{k=1}^{2} \int_{\Delta}\left[\frac{1}{2}\left(1+\sum_{i=1}^{N-1}\left(\frac{\partial y_{k}}{\partial x_{i}}\right)^{2}\right) \frac{\left(\frac{\partial y_{1}}{\partial u}+\frac{\partial y_{2}}{\partial u}\right)}{\frac{\partial y_{k}}{\partial u}}-\sum_{i=1}^{N-1} \frac{\partial y_{k}}{\partial x_{i}}\left(\frac{\partial y_{1}}{\partial x_{i}}+\frac{\partial y_{2}}{\partial x_{i}}\right)\right]\left|\frac{\partial y_{k}}{\partial u}\right|^{-1} d x^{\prime} d u \\
& +o(t) .
\end{aligned}
$$

Using (24), we deduce of (28) that

$$
\begin{aligned}
& \alpha(u, \Omega)=\frac{1}{2} \int_{\Delta}\left(\frac{\partial y_{1}}{\partial u}+\frac{\partial y_{2}}{\partial u}\right)\left(\left(\frac{\partial y_{1}}{\partial u}\right)^{-2}-\left(\frac{\partial y_{2}}{\partial u}\right)^{-2}\right) \\
& +\left(\frac{\partial y_{2}}{\partial u}-\frac{\partial y_{1}}{\partial u}\right)\left(\sum_{i=1}^{N-1}\left(\frac{\frac{\partial y_{1}}{\partial x_{i}}}{\frac{\partial y_{1}}{\partial u}}-\frac{\frac{\partial y_{2}}{\partial x_{i}}}{\frac{\partial y_{2}}{\partial u}}\right)^{2}\right) d x^{\prime} d u .
\end{aligned}
$$

Since each above quantity is non positive, $\alpha(u, \Omega)$ would be zero if and only if $\frac{\partial\left(y_{1}+y_{2}\right)}{\partial u}=0$ and $\frac{\partial\left(y_{1}+y_{2}\right)}{\partial x_{i}}=0$ a.e. $\forall i=1, . ., N-1$, this is only possible if $\Omega$ is symmetric which completes the proof. 
Now, we can conclude for the proof of theorem 2 :

let us denote by $u_{t}$ the first (normalized) eigenfunction on $\Omega_{t}$ (which is - a priori - different from $\left.u^{t}\right)$. Since $u^{t}$ belongs to $H_{0}^{1}\left(\Omega_{t}\right)$, the definition of $\lambda_{1}\left(\Omega_{t}\right)$ gives :

$\lambda\left(\Omega_{t}\right)=\int_{\Omega_{t}}\left|\nabla u_{t}\right|^{2} d x . \leq \int_{\Omega_{t}}\left|\nabla u^{t}\right|^{2} d x$.

Now we can apply lemma 1 to $u$ since the level sets of the first eigenfunction of a convex domain are convex (see Kawohl (1985)), so (30) and (23) yield

$\lambda\left(\Omega_{t}\right) \leq \int_{\Omega}|\nabla u|^{2} d x+\alpha(u, \Omega) t+o(t)$.

So, we obtain

$\frac{\lambda\left(\Omega_{t}\right)-\lambda(\Omega)}{t} \leq \alpha(u, \Omega)+o(1)$

and passing to the limit $\quad d \lambda(\Omega ; V) \leq \alpha(u, \Omega)<0 \quad$ what is the desired result since the Steiner symmetrization field preserves the volume.

\section{REFERENCES}

Brock, F. (1995) Continuous Steiner Symmetrization. Math. Nachrichten, 172, 25-48.

Brock, F. and Henrot, A. (to appear) A symmetry result for overdetermined boundary value problem using domain derivative and continuous Steiner symmetrization.

Chatelain, T. and Henrot, A. (work in progress).

Cox, S.J. (1994) Extremal eigenvalue problems for the Laplacian. M. A. Herrero, E. Zuazua eds, R.A.M. J. Wiley and Masson, 1994.

Kawohl, B. (1985) Rearrangements and Convexity of level sets in Partial Differential Equations. Springer Lecture Notes in Math., 1150.

Mignot, F., Murat, and Puel, J.P. (1979) Variation d'un point de retournement par rapport au domaine. Comm. in p.d.e., 4, 11, 1263-1297.

Rellich, F. (1940) Darstellung der eigenwerk $\Delta u+\lambda u$ durch ein randintegral. Math. $Z$., 46, 635-646.

Rousselet, B. and Chesnais, D. (1990) Continuité et différentiabilité d'éléments propres: application à l'optimisation de structures. Appl. Math. and Optim., 22, 27-59.

Sanchez-Hubert, J. and Sanchez-Palencia, E. Vibration and coupling of continuous systems: asymptotic methods. Springer-Verlag.

J. Serrin, J. (1971) A symmetry problem in potential theory, Arch. Rat. Mech. Anal., 43, 304-318.

Simon, J. (1980) Differentiation with respect to the domain in boundary value problems. Num. Funct. Anal. Optimiz., 2 (7,8), 649-689.

Sokolowski, J. and Zolesio, J.P. (1992) Introduction to shape optimization: shape sensitivity analysis. Springer Series in Computational Mathematics, vol 10, Springer.

L. Zalcman, L. (1992) A bibliographical survey of the Pompeiu problem, in Approximation by solutions of p.d.e., B. Fuglede et al. eds. , Kluwer. 